\title{
Cooperative sensing under limited band control channel in cognitive radio networks
}

\begin{abstract}
In the context of cognitive radio the cooperative spectrum sensing has been proposed to overcome the problem associated with the local sensing for example the hidden node problem due to noise uncertainty, fading, and shadowing. However, sensing results reporting requires perfect channel to avoid the performance degradation. It also requires a large bandwidth under the hypothesis of large number of cognitive users. This paper presents a hard decision auto-correction reporting scheme that is able to correct the error in the reported bit directly. This system further minimizes the average number of the reporting bits by allowing only the user with detection information (binary decision 1) to report its result. The sensing performance is investigated and the numerical results show significant decrease in reporting bits without affecting the sensing performance.
\end{abstract}

Keyword: Cognitive radio; Cooperation; Spectrum sensing 